\title{
A MICROSCOPIC STUDY OF PULPS FROM INFECTED TEETH
}

\author{
AR'THUR T. HENRICI AND THOMAS B. HARTZELL' \\ Department of Bacteriology and Immunology, University of Minnesota, \\ Minneapolis, Minnesota \\ CONTENTS
}

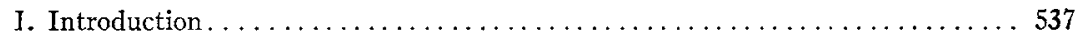

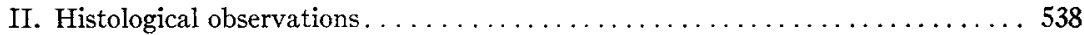

Descriptions of the vital teeth, with statements of the corresponding findings 539

III. Discussion of the findings . . . . . . . . . . . . . . . . . . . . . . 547

[V. General conclusion. . . . . . . . . . . . . . . . . . . . . . . . . . . . . . . . 549

V. Literature cited. . . . . . . . . . . . . . . . . . . . . . . . . . . 550

\section{INTRODUCTION}

It is generally considered that so long as the pulp is protected by an intact wall of dentin, it is free from infection; that only when the pulp chamber becomes exposed by caries or trauma does the pulp tissue become invaded by microörganisms, and that then it usually undergoes suppuration or gangrenous inflammation; and that the pain which occurs previous to actual exposure of the pulp is due to hyperemia, caused either by temperature changes or by lactic acid absorbed through the dentinal tubules. This conception of the process of natural devitalization finds general expression in the text-books on dental pathology.

We believe, on the contrary, that the pulp is frequently invaded by microörganisms long before it has been exposed by caries or, indeed, in the abs'nce of caries, by organisms which have passed into the pulp from the gingival region; and that such invading organisms give rise in the pulp to localized areas of inflammation which are not necessarily suppurative or gangrenous in nature, but rather granulomatous and progressive, leading to fibrosis or other changes. When suppuration and gangrene finally occur, it is in a tissue which has

1 Aided by a grant from the Research Commission of the National Dental Association. 
long been subjected to injury by milder types of infection, and suppuration is here merely the final stage of the process.

The idea that the pulp is sterile until actually exposed by caries is based on a false conception of caries itself, which, however, is still generally taught, namely, that caries is caused by a variety of saprophytic, acid-producing, bacteria and that lactic acid, and a resultant softening, precede bacteria in the invaded dentin. But, as we have pointed out previously, it has long since been demonstrated by Goadby, Kantorowicz, and Niedergesäss (each working independently), that streptococci may be demonstrated, in the dentin, well in advance of any softening; and that, as a matter of fact, saprophytic bacteria and lactic acid are merely the camp-followers of an invading army of pathogenic cocci. It is, therefore, not surprising that the presence of organisms may be demonstrated in the dental pulp long before the softening of the dentin has extended as far as the pulp chamber. In a previous paper (1) we presented the results of bacteriological studies, which indicate that in nearly one-half the number of cases where teeth or their investing tissues are infected, bacteria, of which non-hemolytic streptococci are the predominant species, are already present in the pulp tissues. In concluding that paper we stated that the presence of these bacteria in the pulp "must be accompanied by some chronic inflammatory changes in the tissues." In the work here reported we have made a histological study of a similar series of pulps, and have succeeded in demonstrating the occurrence of the chronic inflammatory reactions which we predicted.

\section{HISTOLOGICAL OBSERVATIONS}

A series of fifty vital teeth, taken as they came from the clinic, were used in this study. They were cracked open with bone-cutting forceps, and dropped into Zenker's solution. After the pulps had been sufficiently hardened, they were carefully stripped out of the pulp chambers and embedded in paraffin. Sections were stained with hematoxylin and eosin. With six specimens, serial sections of the entire pulp were cut. An examination of these sections indicates that, if the same procedure had been adopted for the entire series, a considerably larger number of lesions would have been found. From a 
number of specimens, sections were also stained by the Gram-Weigert method for bacteria, but with negative results.

As in our previous study, all these teeth (with the exception of tooth no. 33, noted in the protocols below) were clinically vital, with pulps presenting neither discoloration nor odor on opening the pulp chamber, and without actual extension of caries to the pulp chamber. From six specimens, owing to technical difficulties, satisfactory sections were not obtained. Three teeth were free from caries or pericemental lesions, and these presented histologically normal pulps. Of the remaining forty-one teeth, thirteen showed pyorrhea alone, five were invaded by caries without pyorrhea, and twenty-three exhibited evidences of both caries and pyorrhea. As in our previous paper w.e include, under caries, teeth with filled cavities where the decay has apparently been arrested.

Descriptions of the fifty vital teeth we studied, and statements of findings relating to them individually, are given below.

Descriptions of the vital teeth, with statements of the corresponding findings

1. Cuspid. Caries; pyorrhea. Pulp normal.

2. Cuspid. Caries; pyorrhea. Pulp normal.

3. Incisor. Caries; pyorrhea. Section shows a small area of subodontoblastic infiltration, with lymphocytes, with some congestion, and two small pulp nodules; also an area of diffuse lime-salt infiltration near the root tip, with dense fibrous tissue in this area.

4. Incisor. Caries; pyorrhea. The pulp has undergone complete fibrosis, all of the connective tissue being of the adult type. A number of small masses of lime salts are scattered throughout the pulp. There is no cellular infiltration.

5. Third molar. Filled caries; pyorrhea. Abscess between the roots. Microscopic examination of the pulp shows fibrosis of the radicular portion and numerous areas of fatty tissue in the coronal part. Three large pulp nodules occupy an intermediate area. There is no inflammatory infiltration.

6. Molar. Caries; no pyorrhea. Section of the pulp shows an area of diffuse lymphocytic and plasma-cell infiltration in one cornu, with edema and congestion in this portion. Otherwise the pulp appears normal. (See figs. 2 and 3.)

7. Molar. Apparently normal. Pulp appears normal under the microscope. 


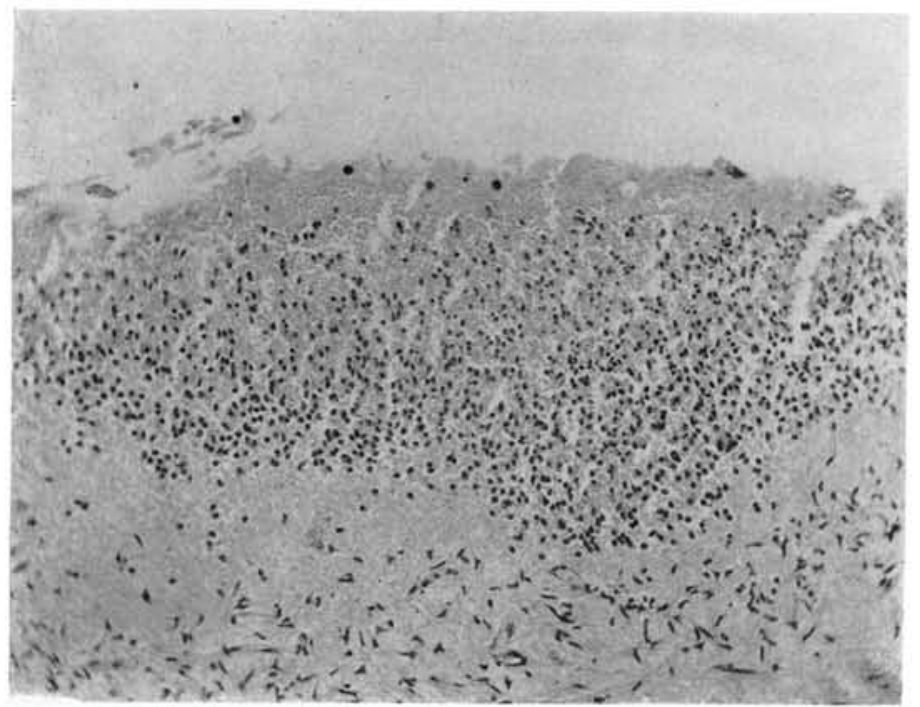

Fig. 1. An Area of Subodontoblastic Inflitrition witi Lymphocytes. (Tooth ํ. 36)

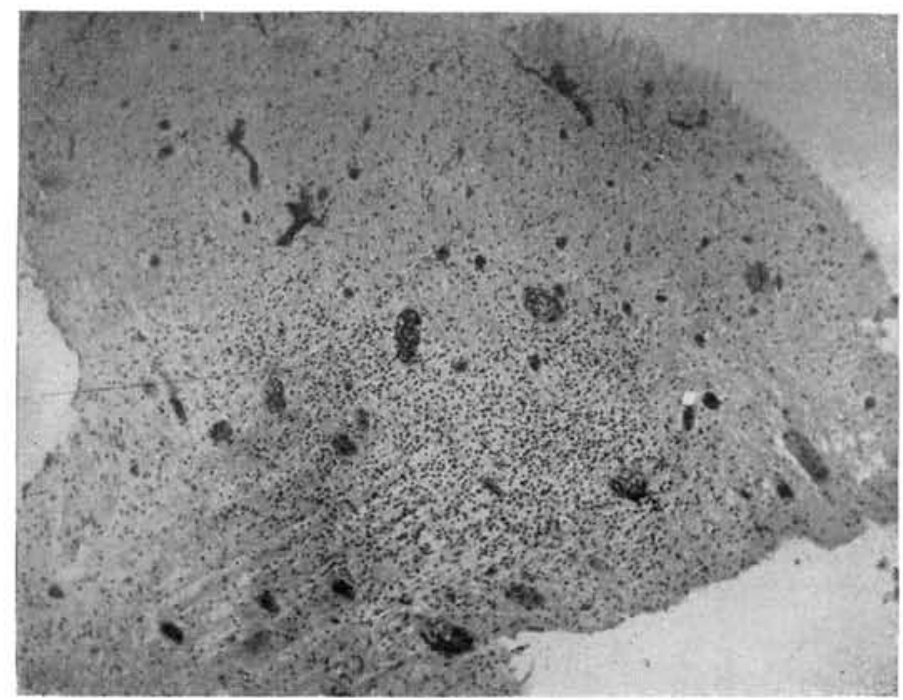

Fig. 2. An Area of Inflammatory Infitration and Congestion in One of the Cornua of a Molar. (Tooth no. 6) 
8. Incisor. Caries; pyorrhea. There is an area of fatty tissue in the coronal portion on one side, extending half-way down the pulp; below this is an area of infiltration, with lymphocytes and a few plasma cells extending from the odontoblastic layer inward for one-third of the thickness of the pulp. There are some heavy fibrous bands and one small calcareous nodule in the radicular portion. The pulp is congested.

9. Molar. Pyorrhea; no caries. Fatty change in one cornu; otherwise normal.

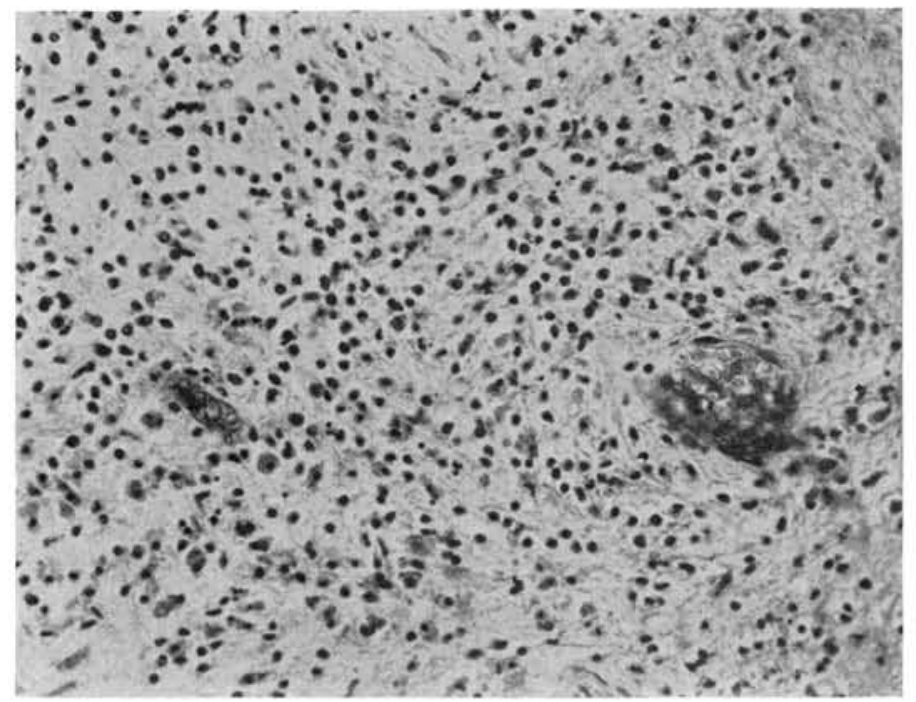

Fig. 3. A Portion of the Last Preceding ( fig. 2), More Highly Magnified, Showing the Monontclear Character of the Inflammatory Celis.

(Тоотн мо. 6)

10. Canine. No caries; no pyorrhea. Pulp normal.

12. Molar. Extensive caries; no pyorrhea. Section of the pulp shows an area of diffuse lymphocytic infiltration beneath the odontoblastic layer; otherwise normal.

13. Molar. Extensive caries; pyorrhea. Section of the pulp shows nothing abnormal.

14. Canine. Pyorrhea; no caries. Pulp normal.

16. Cuspid. Silver filling; some pyorrhea. One small area of lymphocytic infiltration in the coronal portion of the pulp; otherwise normal. 
17. Molar. Extensive filled caries; slight pyorrhea. The pulp shows general fibrosis, dense connective tissue being found in all parts of the section. There is one large irregular calcareous mass in the coronal portion, and a large area of lymphocyte and plasma-cell infiltration beneath the odontoblastic layer; a small, more circumscribed, area occurs internally to the preceding. Some endothelial cells are present in these areas.

18. Molar. Artificial crown. Pyorrhea. Pulp appears normal.

19. Molar. Severe pyorrhea, crown much worn, no caries. Pulp is composed of dense fibrous tissue; infiltration in many areas with lime salts.

21. Molar. Caries; pyorrhea. There is hyperplasia of odontoblasts in the coronal portion of the pulp, the odontoblastic layer being in places four or five cells deep. There are several large irregular calcareous masses and one small circumscribed area of lymphocytic infiltration, in this portion. The radicular portion is composed of dense fibrous tissue, diffusely infiltrated with lime salts.

22. Canine. Pyorrhea; no caries. There is a large amount of fatty tissue in the coronal portion; otherwise the pulp is normal.

23. Canine. Slight caries; pyorrhea. Pulp normal.

24. Molar. Caries; pyorrhea. Pulp normal.

25. Incisor. Large filled cavity. There is considerable fatty tissue, also fibrosis, in the coronal portion; otherwise the pulp is normal.

26. Incisor. Severe pyorrhea. Small filled cavity. Pulp normal.

27. Incisor. Severe pyorrhea. Small filled cavity. The pulp shows general fibrosis, with areas of fatty tissue. The odontoblastic layer in the coronal portion is thickened and there is some lymphocytic infiltration beneath it.

28. Incisor. Severe pyorrhea. Small filled cavity. Pulp normal.

30. Molar. Filled caries; pyorrhea. Pulp normal.

31. Molar. Normal. Pulp shows no change.

32. Molar. Filled caries. "Floating in pus." Section of the pulp shows extensive fibrosis and fatty change; diffuse subodontoblastic infiltration with lymphocytes and plasma cells; some small irregular calcareous nodules in radicular portion.

33. Canine. Caries, extending to the pulp chamber. No pyorrhea. Grossly the pulp appears to be normal in the lower part, but a small drop of pus occupies the upper part of the pulp chamber.

Microscopically, the coronal portion of the pulp shows necrosis and infiltration with polymorphonuclear leucocytes; just beneath this is an area of intense congestion. As we further approach the root, there is found a large area densely infiltrated with plasma cells, and large endothelial 


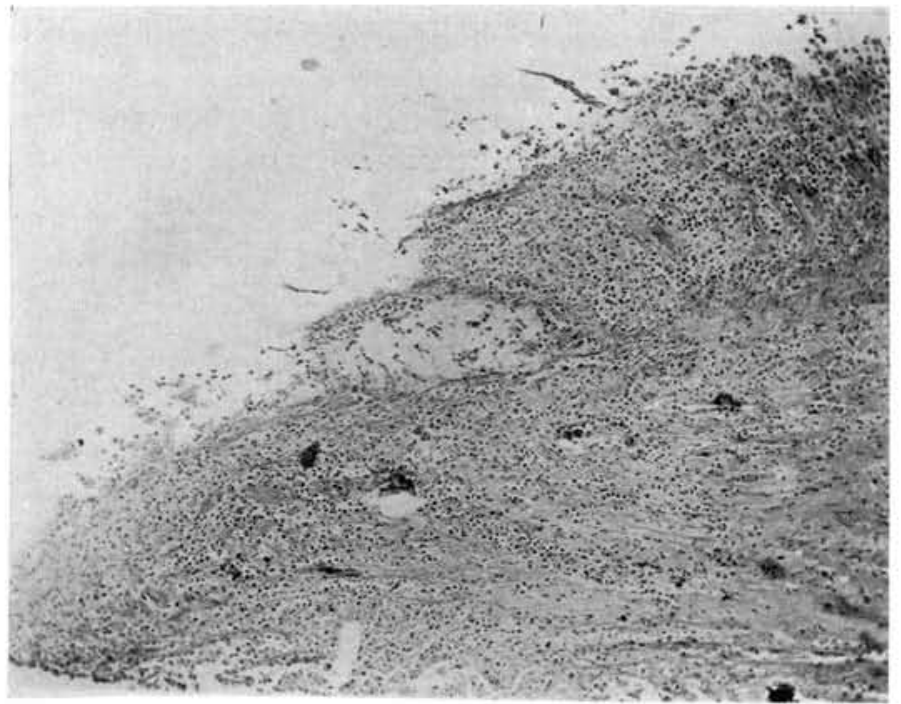

Fig, 4. An Area of Necrotic Tissue with Polymorphonuclear Leucocytes; Coronal Portion. (Tooth No. 33)

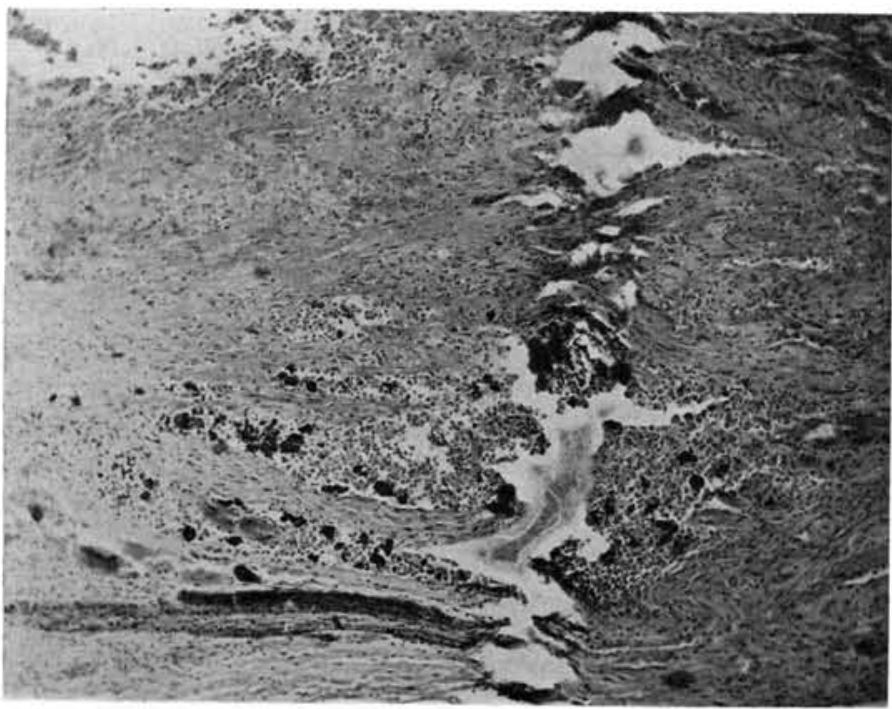

Fig. 5. Small Masses of Lime Salts in a Granulomatous Area. (Tooth no. 33) 


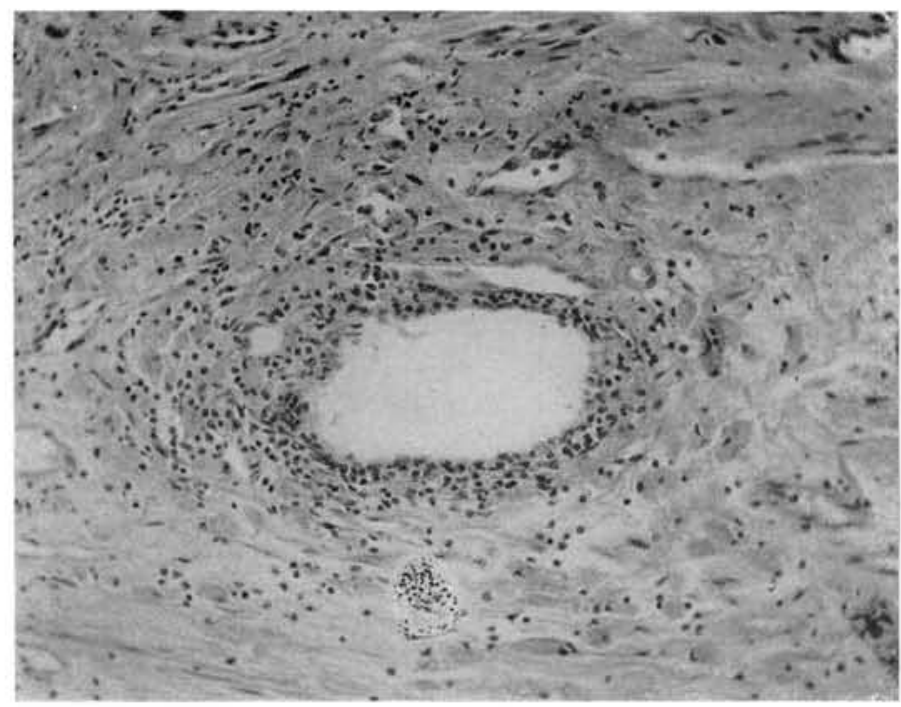

Fig. 6. Perivascular Infiltration with Lymphocytes and Plasma Cells, Surrounded by a Fibroblastic Reaction, (Tooth no. 33)

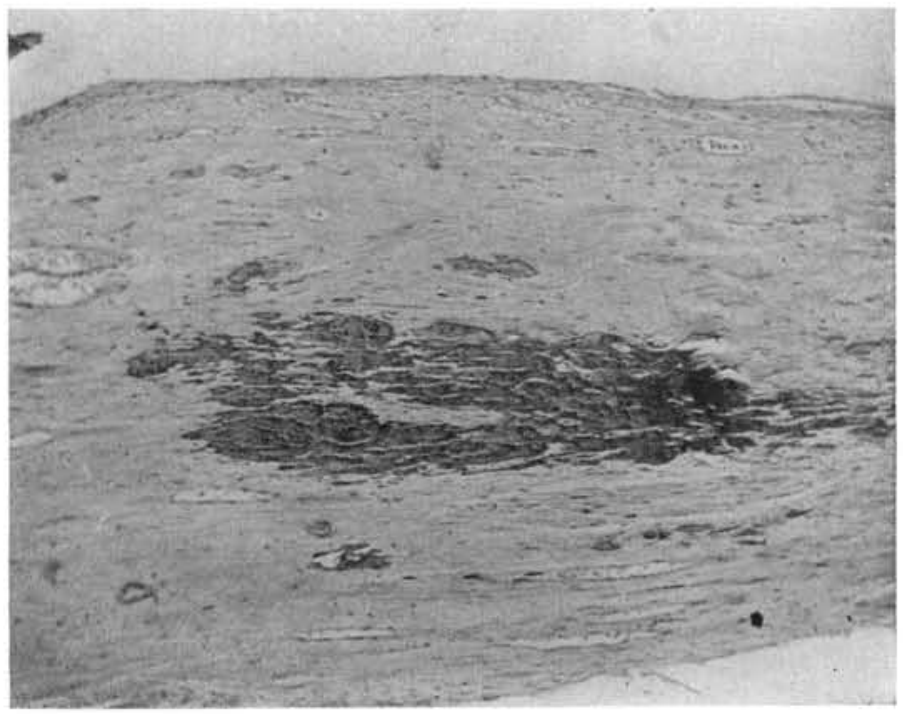

Fig. 7. Calcified Fibrous Tissue in the Radicular Portion. (Tooth no. 33) 
phagocytes, which spread apart the fibrous bundles; several amorphous masses of lime salts are scattered through this area. The connective tissue of the radicular portion is dense and fibrous; one large vessel is surrounded by a zone of lymphocytes and plasma cells, and nearby there are nests of proliferating fibroblasts. As we approach the root tip, the pulp tissue shows extensive diffuse infiltration with lime salts. (See figs. 4, 5,6 and 7.)

(Since the pulp chamber had already been opened by caries, this tooth should not be included in the series proper. The description and corresponding photomicrographs are included in this paper because this one pulp shows all stages in the process of natural devitalization.)

34. Incisor. Slight caries; pyorrhea. Pulp normal.

36. Molar. Pyorrhea; caries; abscess between the roots. One cornu of the pulp shows an extensive area of subodontoblastic infiltration with lymphocytes and plasma cells. There is general fibrosis of the pulp with fatty change. There are several large irregular masses of lime salts in the coronal portion and two distinct pulp nodules in the root. (See fig. 1.)

37. Molar. No caries; pyorrhea. Pulp normal.

38. Molar. No caries; pyorrhea. The coronal portion of the pulp shows a very extensive diffuse infiltration with lymphocytes, plasma cells, and occasional polymorphonuclear leucocytes. The pulp shows general fibrosis, with one large pulp nodule and a number of small irregular calcareous masses. (See figs. 8 and 9.)

39. Molar. Large lateral cavity; no pyorrhea. The pulp shows several small areas of lymphocytic infiltration in the coronal portion. There is some fibrosis, and a few small irregular masses of lime salts are found.

40. Molar. No caries; pyorrhea. Pulp normal.

41. Molar. No caries; pyorrhea. Several small irregular calcareous masses are found in the pulp.

42. Molar. No caries; pyorrhea. The pulp is completely fibrosed and extensively infiltrated with lime salts.

44. Incisor. Pyorrhea; no caries. The pulp consists of a small amount of dense fibrous tissue enclosing numerous large irregular masses of lime salts. The pulp is almost completely calcified.

45. Incisor. Pyorrhea; no caries. The pulp shows some small irregular calcareous masses but is otherwise normal.

46 Molar. Large filled cavity; pyorrhea. There is one large pulp nodule in the coronal portion and extensive calcification in the radicular portion; no inflammatory reaction but some fibrosis.

47. Molar. Pyorrhea; no caries. Pulp normal.

48. Incisor. Filled cavities; pyorrhea. Pulp normal. 


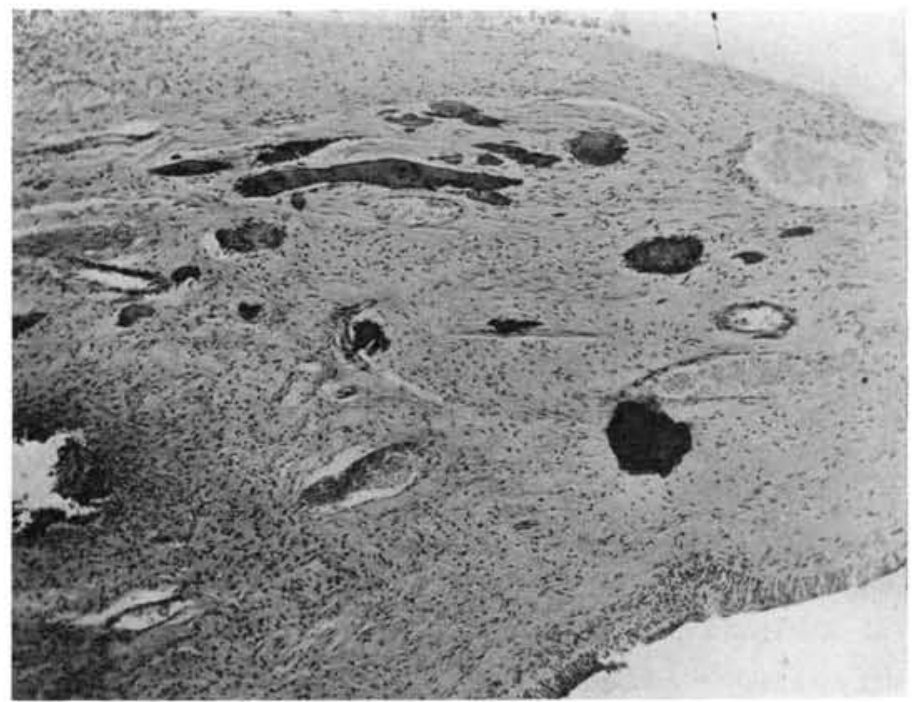

Fig. 8. Islands of Calcified Material Imbedded in Dense Fibrous Tissue. (ТоoтH No. 38 )

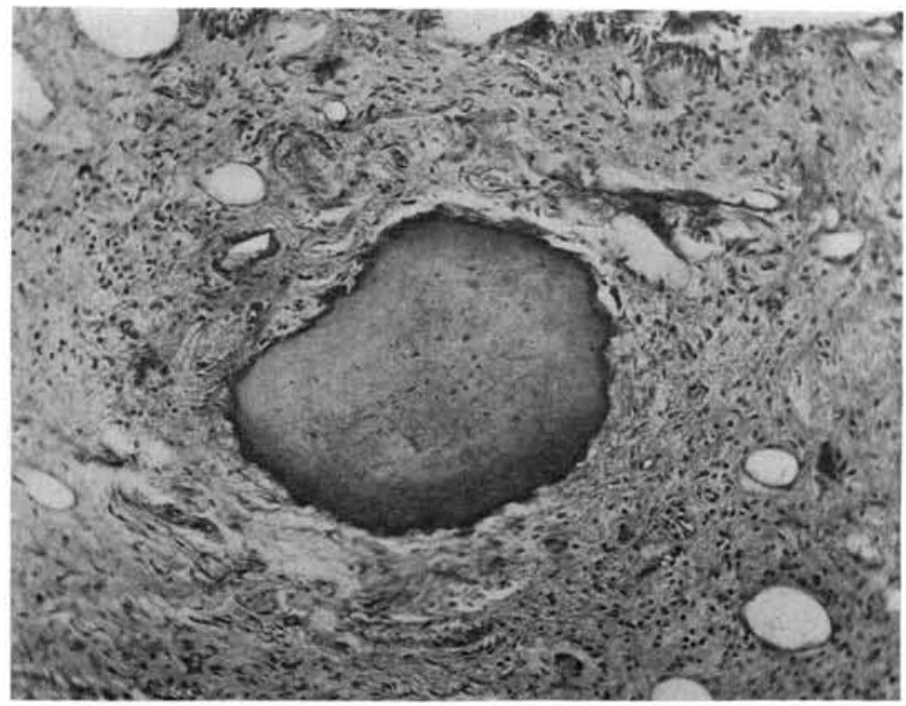

Fig. 9. A Pulp Nodule in Dense Fibrous Tissue. (Tooth no. 38) 
49. Incisor. Filled cavity; pyorrhea. Pulp normal.

50. Incisor. Filled cavities; pyorrhea. At one point there is an area of lymphocytic infiltration in the coronal portion, with fibrosis and fatty change throughout the pulp.

\section{DISCUSSION OF THE FINDINGS}

The lesions observed fall naturally into two classes, namely, (1) inflammatory cellular infiltrations, and (2) secondary or degenerative changes, including fibrosis, calcifications, and fat accumulations. The first alone, of course, can be considered as direct evidence of bacterial activity.

The inflammatory lesions can be best characterised as "miliary granulomata," since they are very small (not visible to the naked eye), usually rather sharply circumscribed, are accompanied by very little destruction of tissue, and exhibit only mononuclear leucocytes, namely, lymphocytes and plasma cells, such as we are accustomed to associate with progressive chronic inflammations in other parts of the body. In only two cases have we found polymorphonuclear leucocytes; in one of these, as noted, the pulp was exposed, and in the other the pus cells were very much in the minority.

These lesions, save for their limited extent, are practically identical with the earliest lesions in the subgingival region in pyorrhea, and the periapical tissues in root "abscesses," as we have described them in a previous paper (2). They are also identical with the majority of the lesions which occur in rabbits experimentally inoculated with dental streptococci. Miliary granulomata were found most frequently in the coronal portion of the pulp, usually just beneath the odontoblastic layer, but also at times free in the pulp tissue, especially in the cornua of molars.

The various lesions which we have included under the heading of secondary or degenerative changes have been well described before, especially by Hopewell-Smith, who ascribed them to a variety of general causes, but especially to senescence. Unfortunately, we have failed to keep a record of the ages of the patients from whom these teeth were extracted, and the series studied is too small to allow us to follow all of these changes in their natural sequence. Nevertheless, we rather believe that these changes are the result of the inflammatory 
reactions just described, and that they represent repeated lesions of a granulomatous character which have undergone spontaneous healing. It is self-evident that the teeth of elderly individuals are more likely to have suffered from repeated infections than those of younger persons.

Fibrosis of the pulp is readily recognized, since the connective tissue of the pulp is normally of an embryonic or mucoid character. We have set down, as fibrotic, all pulps showing any considerable amount of collagen with connective tissue cells of the adult type; although many of the pulps showed a few strands of adult white fibrous tissue in the radicular portion without further change, and were not included as fibrosed.

Fibrosis, as is well known, is a sequel of inflammation wherever it occurs. It is the final stage of tubercle and gumma, of the submiliary nodules of rheumatic myocarditis, and of all granulomatous inflammations which undergo healing. Even the fibroses of appendix and tonsil, of breast and prostate, formerly considered as degenerative changes accompanying senescence, are now generally considered rather as the result of repeated inflammatory reactions, due to bacteria, to which these tissues have been subjected during life. We feel sure, therefore, that the fibroses we have observed in the dental pulp are the scars of previous granulomatous inflammations produced by microörganisms,

The extensive calcifications which occur in the pulp need further study, and an elaborate discussion is beyond the scope of this paper. They have been repeatedly described before, and have been explained in various ways. They have been considered as an aberrant secretion of the dentinogenetic cells, analogous to the callus formed by injured bones; but this is evidently not the case, since we have found them most frequently far from the odontoblastic layer surrounded by adult connective tissue. In fact, in most cases the lime appears to be deposited in the connective tissue fibers themselves. Again, reasoning by analogy from the pathology of other tissues, we believe that these calcareous deposits are also the result of a previous infection, for they occur frequently in tubercles and gummata. We have previously described similar deposits of lime salts in the connective tissues of the peridental membrane in both pyorrhea and apical granulomata. In this connection we might recall that Rosenow has described a phe- 
nomenon which we have also observed, namely, a rapid and rather extensive infiltration with lime salts in some of the lesions produced in rabbits by inoculation with non-hemolytic streptococci; these occur especially in the voluntary muscles.

The fatty changes which we have observed are not fatty degenerations or infiltrations, but deposits of fatty tissue of an adult type in areas of the pulp tissue, the fat cells appearing as hollow spheres with the nucleus in each pushed to one side, and the tissue presenting the areolar appearance of fat tissue as it occurs in other parts of the body. We have found no description of this condition and cannot explain it. The fat was also found associated with fibrosis, and may represent an attempt to fill the space left in the pulp chamber by the shrinkage of the scar tissue formed there. Deposits of fatty tissue accompany chronic or fibrosing inflammations in other parts of the body; for instance, one frequently finds a sclerosed or obliterated appendix embedded in a fatty meso-appendix, even in lean individuals.

The number of teeth studied is too small to warrant quantitative comparisons, either with the bacteriological studies previously reported or between the various groups in the present series, but we have expressed the results of our study quantitatively in the following table:

\begin{tabular}{|c|c|c|c|c|c|c|}
\hline \multicolumn{2}{|l|}{ IRETH } & \multicolumn{5}{|c|}{ RESOLTS OP HISTOLOGICAL EXAMTNATTON } \\
\hline Condition & $\begin{array}{l}\text { Number } \\
\text { examined }\end{array}$ & Normal & $\begin{array}{c}\text { Inflamma- } \\
\text { tory infil- } \\
\text { trations }\end{array}$ & Fibrosis & $\begin{array}{l}\text { Calcif- } \\
\text { cation }\end{array}$ & Fat \\
\hline Normal. ........... & 3 & 3 & & & & \\
\hline Pyorrhea only.$\ldots \ldots \ldots \ldots$. & 13 & 5 & 1 & • 4 & 6 & 2 \\
\hline Caries only $\ldots \ldots \ldots \ldots \ldots \ldots$ & 5 & 0 & 4 & 3 & 2 & 1 \\
\hline Both caries and pyorrhea ..... & 23 & 6 & 9 & 11 & 9 & 6 \\
\hline
\end{tabular}

IV. GENERAL CONCLUSION

Thus, we have found active inflammation in fourteen pulps from forty-one infected teeth, or 37 per cent-ample confirmation of the opinion, previously arrived at from bacteriological studies, that the pulp may be (a) invaded by microörganisms long before it is actually exposed by caries, and may be (b) repeatedly injured long before it actually undergoes necrosis. 


\section{LITERATURE CITED}

(1) 1919 Henrici, Arthur T., and Hartzelt, Thomas B. The bacteriology of vital pulps. Journal of Dental Research, i, p. 419.

(2) 1917 Henrici, Arthur T., and Hartzell, Thomas B. The microscopic anatomy of chronic periodontitis and the pathogenesis of dental root cysts. Journal of the National Dental Association, iv, p. 1061. 